2014-02-20

\title{
Optically significant particle sizes in
}

\section{seawater}

\author{
Davies, EJ
}

http://hdl.handle.net/10026.1/3055

10.1364/AO.53.001067

APPLIED OPTICS

Optica Publishing Group

All content in PEARL is protected by copyright law. Author manuscripts are made available in accordance with publisher policies. Please cite only the published version using the details provided on the item record or document. In the absence of an open licence (e.g. Creative Commons), permissions for further reuse of content should be sought from the publisher or author. 


\title{
Optically significant particle sizes in seawater
}

\author{
Emlyn J. Davies, ${ }^{1,4, *}$ David McKee, ${ }^{2}$ David Bowers, ${ }^{3}$ \\ George W. Graham, ${ }^{1}$ and W. Alex M. Nimmo-Smith ${ }^{1}$ \\ ${ }^{1}$ School of Marine Science and Engineering, Plymouth University, Devon, UK \\ ${ }^{2}$ Department of Physics, University of Strathclyde, 107 Rottenrow, Glasgow, UK \\ ${ }^{3}$ School of Ocean Sciences, Bangor University, Menai Bridge, Angelsey, Wales UK \\ ${ }^{4}$ Currently at: Department of Environmental Technology, SINTEF: Materials and Chemistry, Trondheim, Norway \\ ${ }^{*}$ Corresponding author: emlyn.john.davies@ sintef.no
}

Received 15 October 2013; revised 19 December 2013; accepted 15 January 2014; posted 16 January 2014 (Doc. ID 199420); published 12 February 2014

\begin{abstract}
Small particles $(<10 \mu \mathrm{m})$ are often considered to play the dominant role in controlling scattering and absorption due to their relatively large numbers, which are typically found in the ocean. Here we present an approach for quantifying the size range of particles that contribute significantly to bulk inherent optical properties. We present a numerical assessment of the variability in optically significant particle sizes for simplistic populations that conform to the assumptions of homogeneous, spherical particles, and power-law size distributions. We use numerical predictions from Mie theory to suggest minimum and maximum particle sizes required for accurate predictions and observations of ocean optics for different particle size distributions (PSDs). When considering observed ranges of PSDs, our predictions suggest the need for measurements of optical properties and particles to capture information from particle sizes between diameters of $0.05-2000 \mu \mathrm{m}$ in order to properly constrain relationships between particles and their associated optical properties. Natural particle populations in the ocean may present more complex PSDs that could be analyzed using the method presented here to establish optically significant size classes. (C) 2014 Optical Society of America

OCIS codes: (290.4020) Mie theory; (290.5850) Scattering, particles; (290.7050) Turbid media; (010.4450) Oceanic optics; (010.4458) Oceanic scattering.

http://dx.doi.org/10.1364/AO.53.001067
\end{abstract}

\section{Introduction}

Marine suspended particles play a key role in controlling the optical properties of seawater. The marine optics literature commonly partitions the particle population into phytoplankton, which are ubiquitous in natural waters, and mineral particles, which are abundant in near-shore regions. Multiple studies, utilizing a range of measurement techniques, have shown marine particles to range in size over several orders of magnitude, from submicron colloids and bacteria to flocculated aggregates and macroplankton, which may reach dimensions of

$1559-128 \mathrm{X} / 14 / 061067-08 \$ 15.00 / 0$

(C) 2014 Optical Society of America the order of millimeters [1-4]. Variability in the origin and type of particles present in the natural environment produces optical properties that are highly changeable through time and space. Accurately relating inherent optical properties (IOPs) to measurements and predictions of particle population characteristics remains an outstanding challenge. A fundamental requirement for understanding the controlling factors in ocean optics is to achieve closure between IOPs and the particle population parameters controlling them, e.g., $[5,6]$.

Successful closure between the properties of particle populations and IOPs first requires an understanding of the size range of particles that are of optical significance. A multitude of approaches exist to represent oceanic particle size distributions 
(PSDs), including (but not limited to) those of Risović [7] and Jonasz and Fournier [8]. The complexities and details of natural particle populations that can be resolved within numerical representations of the PSD are dependent on the quality and reliability of observations. Currently available in situ particle sizing instrumentation does not provide sufficient information to adequately populate some of these more advanced PSD models. It remains the case that the most commonly used approximation for oceanic particle PSDs is the power law (or Junge distribution). Moreover, further simplification by assumption of a typical exponent of -4 , has led to an understanding that optical properties are primarily controlled by particles of between approximately 0.1 and $10 \mu \mathrm{m}$ $([9,10])$, due to a combination of relative abundance and scattering cross section in comparison to particles in other size classes. However, these estimations have accounted for a limited range of PSDs in comparison to the observed variability within the marine environment $[\underline{3}, \underline{4}, \underline{11}]$. Subsequently, optically significant particles sizes are yet to be clearly defined and identified for marine optics over the broad range of relevant size distributions.

Here we present estimated ranges of optically significant particle sizes for simplistic particle populations that cover the variability typical of PSDs observed in situ, with the purpose of formulating a clear understanding of the PSD ranges required in order to establish closure with IOPs and also to inform development of future IOP measurement systems. We specifically limit the analysis to power-law PSDs for simplicity only; this should not be misinterpreted as a suggestion that natural populations follow this distribution. As a first step toward developing an understanding of likely size ranges for optically significant particles, we explore the impact of varying the power-law exponent through a range of values obtained from the literature.

\section{A. Inherent Optical Properties}

Particle shape, composition, and size distribution all influence the interaction of light with the ocean. The parameterization of these three controlling factors leads to the introduction of assumptions about particle populations prior to any optical prediction, whether used in modeling or in observational corrections. The simplistic and commonly used assumption of spherical particles with homogeneous composition permits the use of Mie theory in modeling the angular distribution of scattering intensity [volume scattering function (VSF)] and attenuation for a given particle size and refractive index. This information can then be used to derive the bulk IOPs of scattering $(b)$, backscattering $\left(b_{b}\right)$, attenuation $(c)$, and absorption ( $a$ ) via subtraction of $b$ from $c$. In this paper, we use the assumption of homogeneous spherical particles so that we can exploit simple Mie calculations and focus on size effects. However, we note that natural particle populations present a multitude of complex shapes whose effects are not covered here and would require more complex optical modeling strategies.

Particle size and composition control scattering and attenuation through the well-understood physical interactions between particles and light, which are governed by Maxwell's electromagnetic wave equations and applied more conveniently in Mie theory. However, some of these fundamental controls on scattering are sometimes overlooked and so are emphasized here for clarity. The VSF $(\beta)$ can be calculated by integrating the scattering cross section $\left(C_{\mathrm{sca}}\right)$ and phase function $(\tilde{\beta})$ over the size distribution using Eq. (1) (e.g., [12]):

$$
\beta=\int_{0}^{\infty} N(D) \tilde{\beta} C_{\mathrm{sca}} \mathrm{d} D,
$$

where $N(D)$ is the number of particles of diameter, $D$, per unit volume. The phase function $(\tilde{\beta})$ is computed as per Bohren and Huffman [13].

To illustrate the effect of particle size and composition on scattering, the VSFs for single particles [i.e., $N(D)=1$ ], with diameters ranging from $0.1-$ $100 \mu \mathrm{m}$, are shown for an absolute refractive index $\left(m_{p}\right)$ typical of a nonabsorbing mineral particle $\left(m_{p}=1.55+0 i\right)$, in Figs. $\underline{1(\mathrm{a})}$ and $1(\mathrm{~b})$, and for absorbing plankton $\left(m_{p}=\overline{1.45}+0.0 \overline{01 i}\right.$ [14] $)$, in Figs. 1(c) and 1(d). Figure 1 demonstrates that large particles produce stronger scattering with more peaks and troughs than small particles and that large particle VSFs are more forward-peaked (i.e., have higher intensities at smaller angles) than smaller particles. Once particles reduce to sizes approaching the wavelength of incident light (i.e., within the Rayleigh-dominant size range), the scattering function becomes almost flat, with only a single trough in the side-scattering region $\left(\sim 90^{\circ}\right)$. The comparison of mineral and biological scattering shows the dominant role played by the refractive index at larger angles (and therefore primarily affecting backscattering), in contrast to the diffraction-dominated forward angles [15]. For equivalent particle sizes, mineral particles typically exhibit higher backscattering signals than phytoplankton [as is evident when comparing the $100 \mu \mathrm{m}$ scattering in Figs. 1(a) and 1(c), for example].

Scattering coefficients can be obtained by integrating the VSF $(\beta)$ over all angles $(\theta)$ for total scattering $(b)$ with Eq. (2), and backscattering $\left(b_{b}\right)$ with Eq. (3):

$$
\begin{aligned}
& b=2 \pi \int_{0}^{\pi} \beta \sin (\theta) \mathrm{d} \theta, \\
& b_{b}=2 \pi \int_{\frac{\pi}{2}}^{\pi} \beta \sin (\theta) \mathrm{d} \theta .
\end{aligned}
$$

Figure 2(a) summarizes the increase in total scattering and backscattering coefficients with particle size for mono-disperse mineral particles $[N(D)=1]$, with the associated change in backscattering ratio 

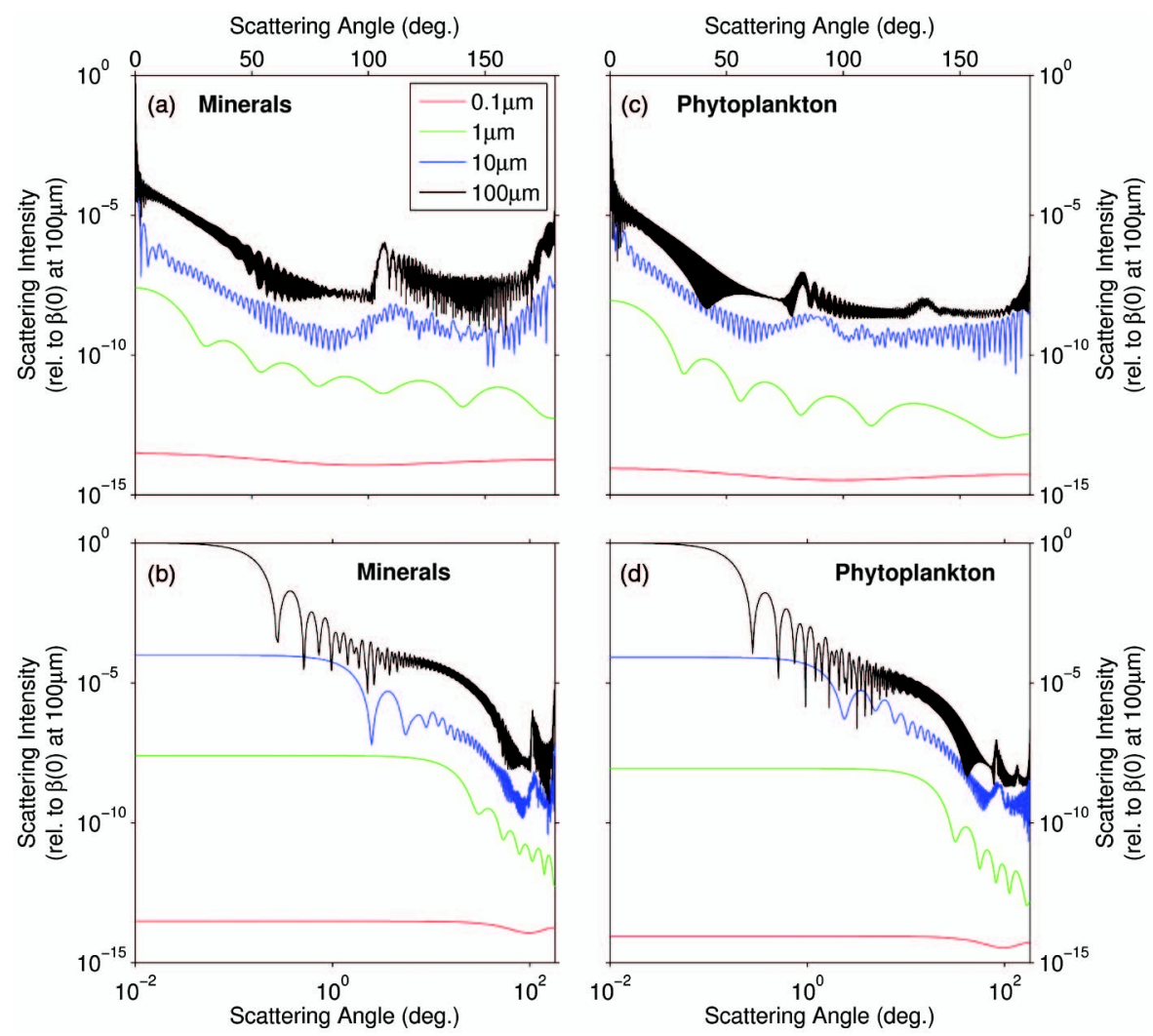

Fig. 1. VSFs, normalized to $\beta(0)$ at $100 \mu \mathrm{m}$ for single-sized particles for a mineral refractive index (a) and (b) of $1.55+0 i$, and a plankton refractive index (c) and (d) of $1.45+0.001 i$. The wavelength of incident light is $532 \mathrm{~nm}$ and refractive index of water is 1.33 . Note the use of linear $x$ axis for (a) and (c) and $\log x$ axis for (b) and (d) to expand the detail of the scattering function for large and small angles.

$\left(b_{b} / b\right)$ shown in Fig. 2(b). It is important that a suitable angular resolution is used when calculating $b$ and $b_{b}$ with Eqs. (2) and (3) because the highest scattering intensities are at very small angles for particles larger than approximately $0.1 \mu \mathrm{m}$. To account for this, the VSF was calculated at the following angles: every $0.001^{\circ}$ from $0^{\circ}$ to $0.5^{\circ}$; every $0.01^{\circ}$ from $0.5^{\circ}$ to $2^{\circ}$; and every $0.1^{\circ}$ from $2^{\circ}$ to $180^{\circ}$. These were selected based on the angular resolutions, above which there was minimal change in the backscattering ratio for particles greater than $100 \mu \mathrm{m}$. Angular resolutions coarser than those used here result in an underestimation of total scattering and an increase in total backscattering ratio for larger particles.

Both total scattering and backscattering coefficients increase rapidly with particle size. Backscattering and forward scattering are equal $\left(b_{b} / b=0.5\right)$ for particles close to the wavelength of incident light (i.e., within the Rayleigh scattering size range). Once sizes increase, and the VSF becomes more forward-peaked, the backscattering ratio is reduced. However, it is interesting to note that as particle sizes increase beyond $1 \mu \mathrm{m}$, and the peaks and troughs of the VSF become increasingly complex (as shown in Fig. 1), so too does the variability in backscattering ratio. It is evident from Fig. 2(b) that
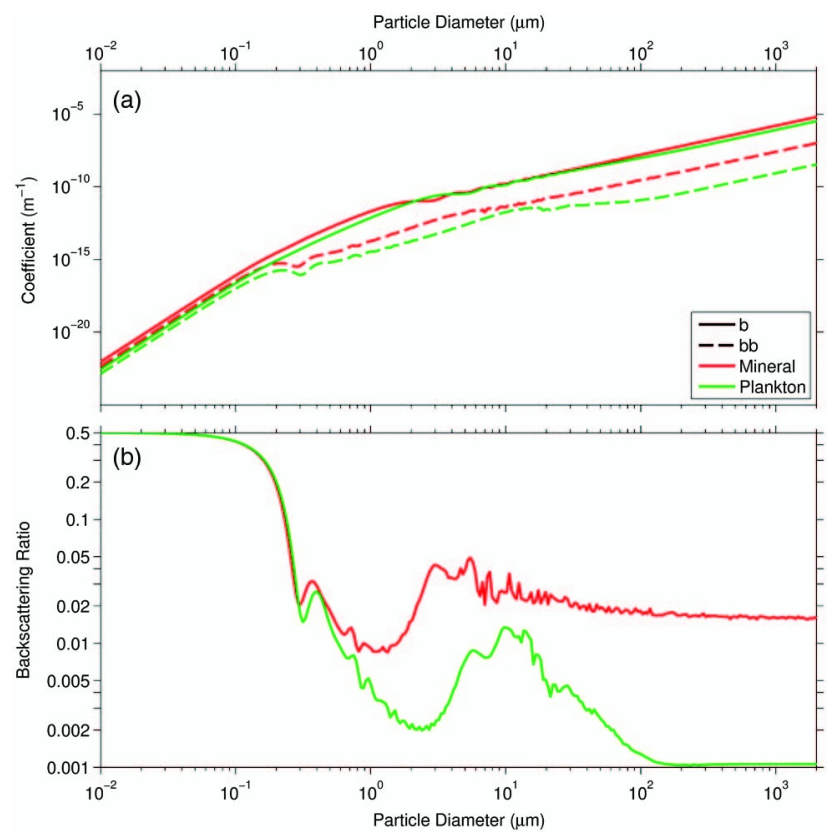

Fig. 2. Scattering and backscattering coefficients for single-sized particles for mineral $\left(m_{p}=1.55+0 i\right)$ and plankton $\left(m_{p}=\right.$ $1.45+0.001 i)$ refractive indices (a), and the associated backscattering ratios (b). The wavelength of incident light is $532 \mathrm{~nm}$, and the refractive index of water is 1.33 . 
there is an increase in backscattering ratio between 2 and $10 \mu \mathrm{m}$ for mineral-type particles and 5 and $20 \mu \mathrm{m}$ for plankton-type particles. For sizes larger than this, backscattering ratios start to converge to about 0.018 for mineral particles larger than about $30 \mu \mathrm{m}$. This coincides with the sizes above which the number of peaks and troughs in the VSF start to increase rapidly. The consequence of this increase in the number of peaks and troughs in the VSF is for the function, $b_{b}(D)$, to oscillate at an ever-increasing frequency and reducing amplitude as sizes increase beyond about $5 \mu \mathrm{m}$. For plankton-type particles, the backscattering ratio reduces to just above 0.001 for large sizes (above $200 \mu \mathrm{m}$ ). It is worth stressing again here that the assumption of homogeneous spheres for larger particles such as this is unlikely to be representative for a natural marine particle population. However, the goal of this article is to quantify optically significant size ranges required to match the most commonly used assumptions of homogeneous spheres conforming to Jungle-like PSDs. The method described in the following sections does, however, remain applicable to more complex particle populations, where scattering and the PSD may be represented in an alternative way.

\section{B. Particle Size Distribution}

The PSD is often assumed to fit closely to a negative power-law distribution (e.g. [11]), which is represented by Eq. ( $\underline{4})$ :

$$
N(D)=K D^{J}
$$

where $N(D)$ is the number of particles of diameter, $D$, per unit volume; $K$ controls the particle concentration; and the (negative) exponent, $J$, controls the relative proportions of small to large particles. Values for the $J$ exponent are often assumed close to -4 . Wide ranges of exponents ( -2.64 to -6.68$)$ have been suggested (e.g., [11]), but the most commonly assumed variability is \pm 1 , which covers the majority of observed ranges reported in the literature (e.g., $[\underline{3}, \underline{4}])$.

Despite the substantially higher scattering intensities for individual large particles in comparison to individual small particles (Fig. 1), the relatively low abundance of large particles in the marine environment [governed by a representative size distribution, such as Eq. (4)], generally results in a maximum size class that contributes significantly to IOPs. The converse is true for small particles, with a minimum size class that is optically significant for a given PSD. Information on these optically significant size ranges has not been well documented for the full range of observed PSD slopes and for the typical ranges of refractive index.

\section{Results and Discussion}

\section{A. Computational and Observational Restrictions}

The VSF should be integrated over the PSD, according to Eq. (1), with $D$ spanning zero to infinity. However, this is not possible in reality; computationally because the number of calculations required for deriving the phase function increases with size; and observationally because large particles are hard to measure in situ without disruption or break up, and small particles are difficult to detect due to their weak and featureless scattering. As a result, both computational and observational studies effectively impose practical limits $\left(D_{\min }\right.$ and $\left.D_{\max }\right)$ on the diameters used for this integration. This leads to the following approximation of the VSF [Eq. (ㅁ)]:

$$
\beta=\int_{D_{\min }}^{D^{\max }} N(D) \tilde{\beta} C_{\mathrm{sca}} \mathrm{d} D \leq \int_{0}^{\infty} N(D) \tilde{\beta} C_{\mathrm{sca}} \mathrm{d} D .
$$

Scattering coefficients $\left(b\right.$ and $b_{b}$ ) will subsequently decrease as $D_{\max }$ reduces or as $D_{\min }$ increases. The VSFs for $D_{\max }$ values of $0.1,1$, and $100 \mu \mathrm{m}$, and a fixed $D_{\min }$ of $0.001 \mu \mathrm{m}$ are shown in Fig. 3 for mineral (a) and plankton (b) particles. The VS $\bar{F}_{s}$ of $<1 \mu \mathrm{m}$ and $<100 \mu \mathrm{m}$ are relatively similar between $20^{\circ}$
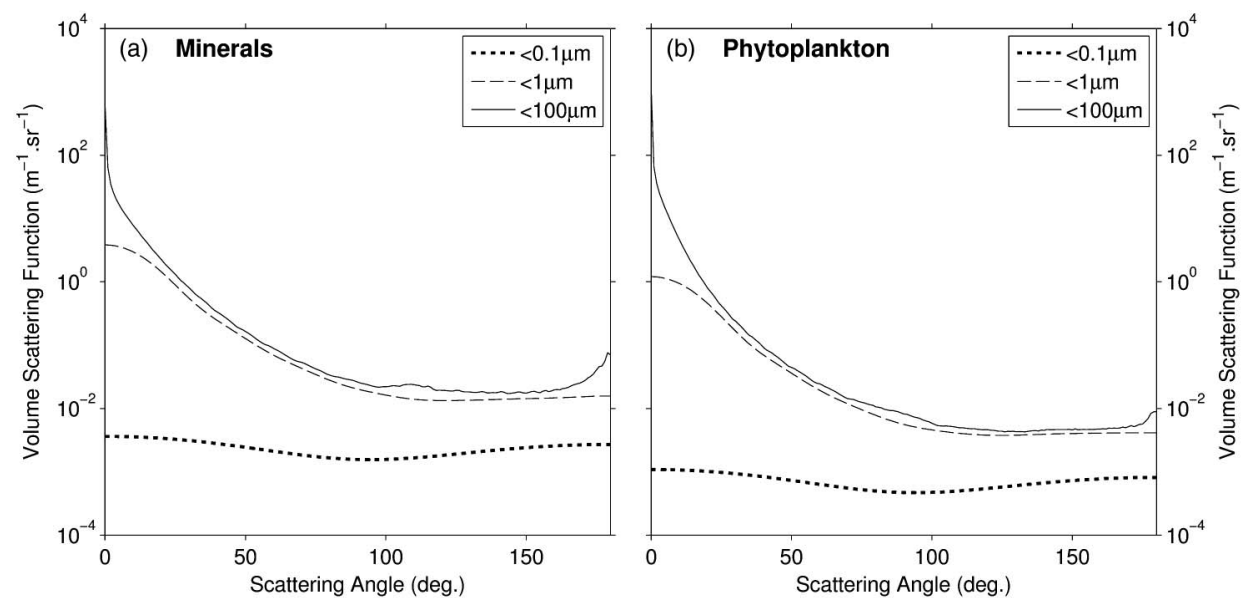

Fig. 3. VSFs for PSDs with $D_{\max }$ values of $0.1,1$, and $100 \mu \mathrm{m}$, and a fixed $D_{\min }$ of $0.001 \mu \mathrm{m}$ for mineral (a) and plankton (b) particles with refractive indices of $1.55+0 i$ and $1.45+0.001 i$, respectively. 
and $160^{\circ}$, with the majority of the change in scattering occurring at angles less than $5^{\circ}$. Because the smaller angles of the VSF are primarily dominated by particle size, forward scattering is much more sensitive to $D_{\max }$ than backscattering. Backscattering is subsequently less changeable, but the comparison of the larger-angle regions of Figs. 3(a) and 3(b) does, again, illustrate the important influence of particle composition on these backscattering angles of the VSF.

It is important to note at this stage that the integration of the VSF over multiple size classes will be sensitive to the resolution of size classes used. For insufficient size class resolution, the VSFs spanning larger size ranges (such as $<100 \mu \mathrm{m}$ ) will become contaminated by the smaller amplitude fluctuations apparent in Fig. 1. The smoothed scattering functions in Fig. 3 for these larger limits are therefore an indication of sufficient particle size resolution.

\section{B. Application to Bulk Inherent Optical Properties}

It has been suggested by Stramski and Kiefer [9] and Babin et al. [10] that the impact that the $D_{\min }$ and $D_{\max }$ integration limits have on bulk IOPs can be assessed by varying $D_{\max }$ until the coefficient converges. However, the approximation of the VSF, shown in Eq. (5), leads to scattering coefficients [Eqs. (2) and (3)] that will never fully converge if $D_{\max }$ is less than infinity. This therefore becomes a problem that cannot be easily rectified, but it is possible to quantify the significance of the integration limits for different particle populations so that future measurements and models can be adapted accordingly. Here $D_{\max }$ is extended beyond the $100 \mu \mathrm{m}$ limit used by Stramski and Kiefer to $5000 \mu \mathrm{m}$, with the assumption that these excessively large diameters, coupled with a $D_{\min }$ of $0.001 \mu \mathrm{m}$, will enable calculations of IOPs that are indistinguishable from their "true" value under the assumptions governed by a power-law distribution. We have therefore restricted our numerical predictions to power-law exponents of up to -3.2 , as gradients shallower than this are uncommon and would require consideration of particle diameters approaching $\mathrm{cm}$ scales. In a realistic marine environment, the largest particles may easily extend to $5000 \mu \mathrm{m}$ (as mineral aggregates, for example), but it is also likely that large particles (e.g., greater than $\sim 250 \mu \mathrm{m}$ ) will not be spherical or homogeneous in composition (e.g., [16]). These deviations from homogeneous spheres, which are especially evident for large particles, have created additional complexities in understanding the optical properties of the water [17] and, in some cases, even posed complications for obtaining seemingly simple measurements of particle size [4]. For clarity, we have therefore restricted the scope of this analysis to consider only the most simplistic cases in order to demonstrate the need to assess the significant particle size ranges, prior to defining the integration limits for Eq. (1).

Total scattering $(b)$ and backscattering $\left(b_{b}\right)$ coefficients have been calculated over varying $D_{\max }$ and a fixed $D_{\min }$ of $0.001 \mu \mathrm{m}$ for mineral and phytoplankton refractive indices and three power-law exponents (Fig. 4). For power-law exponents $(J)$ of -4 (solid line), $\bar{F}$ ig. 4(a) shows rapid increases in total scattering between $D_{\text {max }}$ diameters of about 0.1 and $10 \mu \mathrm{m}$. However, it is evident that, as the power-law exponents become shallower (less negative), the upper limits of $D_{\max }$ play a much more substantial role in controlling total scattering. The effect of refractive index is mostly a horizontal offset in the percentage change to total scattering, as opposed to a change in gradient. This is not the case for backscattering [Fig. 4(b)]. Percentage changes to backscattering that result from different $D_{\max }$ limits are generally more
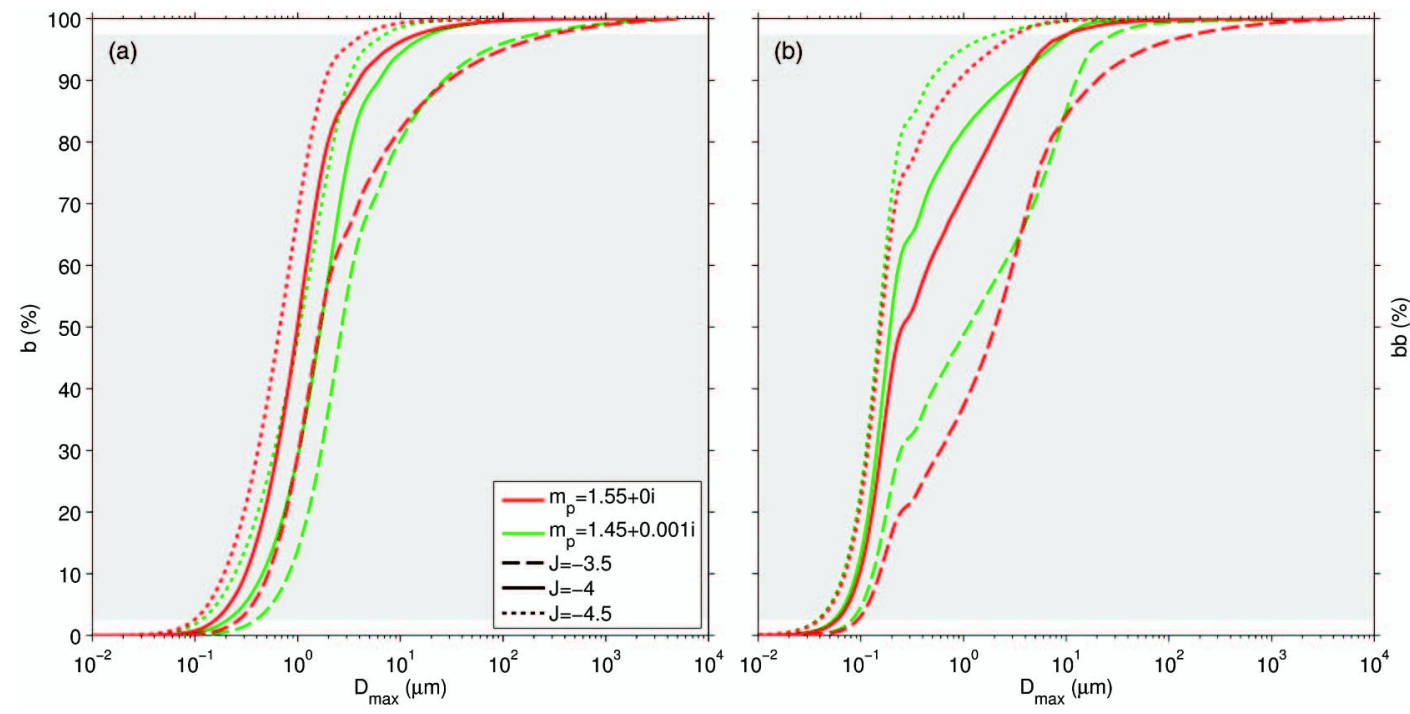

Fig. 4. Cumulative scattering (a) and backscattering (b) for mineral $\left(m_{p}=1.55+0 i\right)$ and plankton $\left(m_{p}=1.45+0.001 i\right)$. Line colors are indicative of refractive index, and the line style indicates the power-law exponent $(J)$. The gray shaded region indicates the areas within the middle $95 \%$ (i.e., from $2.5 \%$ to $97.5 \%$ ) change in scattering and backscattering coefficients. 
complex than the total scattering equivalents. This is due to the high numbers of large particles that are associated with shallow PSD slopes, leading to a greater sensitivity to the backscattering fluctuations identified in Fig. 2. In the small size limits (less than about $0.5 \mu \mathrm{m}$ ), changes in backscattering appear simple [Fig. 4(b)], but the cumulative effects of increasing $D_{\max }$ beyond $5 \mu \mathrm{m}$ and varying refractive index makes predicting the accuracy of backscattering estimates substantially harder.

The extreme sensitivity of backscattering estimates to deviations in power-law exponents highlights the need for caution when attempting to derive total particle concentration from a backscattering signal, as changes in the PSD slope will heavily influence the sizes that dominate the signal received. This is problematic, as accurate calibration of a backscattering signal, to estimate total particle concentration, is reliant on no change in the size distribution or refractive index between calibration and measurement. In the simplest case, where the refractive index is constant and the size distribution conforms to a negative power law [Eq. (4)], backscattering could be calibrated to resolve changes in $K$ only if $J$ was constant (or vice-versa); it would not be possible to isolate the differences that would result from changes in both.

\section{Optically Significant Particle Sizes}

We define "optically significant" sizes as those that account for the middle 95\% change in $b$ and $b_{b}$, respectively (gray region of Fig. 4). Using this definition, we can suggest the ranges of $D_{\min }$ and $D_{\max }$ required to model or observe scattering to an accuracy of up to $95 \%$ in an idealized scenario that meets all the conditions of Mie theory and powerlaw distributions. These calculated limits are shown in Fig. 5 for mineral, nonabsorbing plankton and absorbing plankton particles, over power-law exponents ranging from -4.8 to -3.2 . The use of a nonabsorbing plankton-like refractive index $\left(m_{p}=\right.$ $1.45+0 i$ ) is solely to illustrate, in isolation, the effects of the real and imaginary parts of the refractive index.

It is evident from Figs. 4 and 5 that substantially higher values of $D_{\max }$ are required than previous studies have suggested. This is because studies such as Stramski and Kiefer [9] and Babin et al. [10], considered power-law exponents that were close to -4 . In reality, these slopes may, in some cases, drop below $-3[3,4,11]$.

Figure $5(\mathrm{a})$ shows that for steep power-law exponents $(J=-4.8)$, the range of diameters required for total scattering is relatively narrow and centered around small diameters, with minimum and maximum sizes of 0.1 and $5 \mu \mathrm{m}$, respectively. There is little change that results from absorption (represented by a change in the imaginary refractive index of 0 to $0.001 i$ ), but the difference in the real part of the refractive index (between 1.55 and 1.45) does cause a change of $4 \mu \mathrm{m}$ in the maximum size for this powerlaw exponent. All three particle compositions require a shift in the size range toward larger particles as the power-law exponent becomes less negative, but with the mineral refractive index being less sensitive to this until exponents reach -3.8 . For $J=-4$, the significant size range lies between about 0.2 and $30 \mu \mathrm{m}$. For exponents that are shallower than -4 , the upper size limit required increases rapidly until the point where, for slopes beyond -3.2 , it would be difficult to accurately quantify total scattering because the influence of $\mathrm{mm}$ scale particles becomes relevant.

For backscattering [Fig. 5(b)], the changes in the significant size ranges over different power-law slopes conform to a similar pattern to that of the total scattering, although with the minimum required size shifted to the smaller diameters of 0.03 and $0.15 \mu \mathrm{m}$ for exponents of -4.8 and -3.2 , respectively.
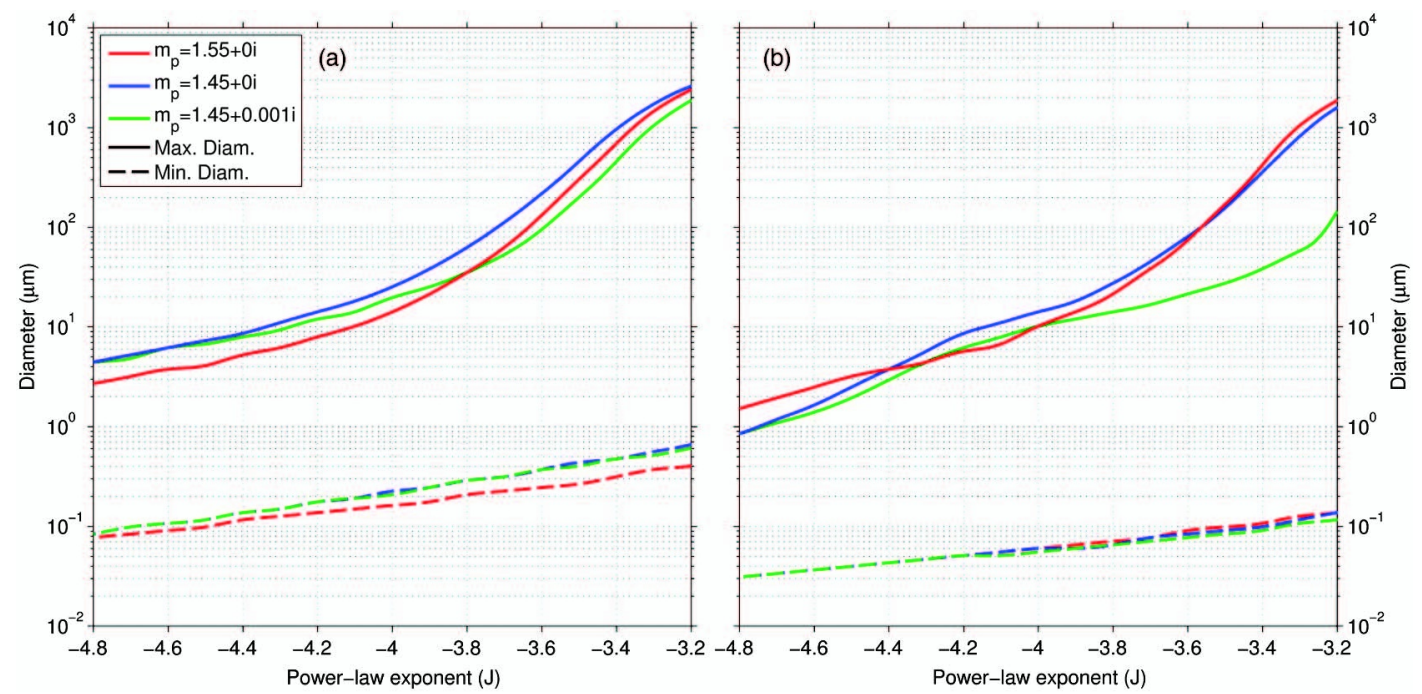

Fig. 5. Estimations of $D_{\min }$ and $D_{\max }$ required to account for up to $95 \%$ of total scattering (a) and backscattering (b) for mineral $\left(m_{p}=1.55+0 i\right)$, nonabsorbing plankton $\left(m_{p}=1.45+0 i\right)$, and absorbing plankton $\left(m_{p}=1.45+0.001 i\right)$ refractive indices. Line colors are indicative of refractive index; line style indicates the minimum and maximum significant diameters, respectively. 
The second most notable difference between the maximum sizes required for backscattering, in comparison to total scattering, is that absorption (represented by a complex refractive index of 0.001 ) causes a substantial decrease in the maximum significant diameter (in relation to a nonabsorbing equivalent) for power-law slopes that are less negative than -3.8 .

The relatively large and variable ranges of particle sizes required to accurately represent IOPs should be a fundamental consideration in formulating observational and numerical methods in ocean optics. While the assumption of power-law exponents of -4 is convenient, it is imperative that any potential for deviations on either side of this are considered, as only small reductions in the gradient of the size distribution can substantially increase the maximum diameters required for optical closure. Caution should also be applied to interpretation of measured and calculated optical properties, as these bulk parameters could be disproportionally affected by specific particle sizes. Substantial changes in bulk optical coefficients could be brought about via alterations in refractive index or the shape of the size distribution, in addition to the particle concentration. It is therefore important to consider the impact of any change to particle composition or size distribution prior to any interpretation of a scattering or backscattering coefficient.

For power-law populations with a more complex compositional makeup, the estimations of $D_{\min }$ and $D_{\max }$ made here may be optimistic. Due to the potential for more specific scenarios to create highly unique optical properties (for example, a phytoplankton bloom), we can only recommend that the particle population should be characterized accurately enough-whether through an alternative numerical representation (e.g., [7]), through more complex use of refractive index (e.g., [16-18]), or through direct in situ observations (e.g., $[3,4])$ - to conduct a similar assessment of the optical significance of each component of the population and reduce the potential for misinterpretation of the optical signals.

\section{Conclusions}

Scattering and backscattering coefficients can be estimated with measurements or models. However, these estimations are affected by restrictions on the particle size ranges that are effectively included, either through observational constraints or numerical convenience. The impact of these assumptions has been assessed in this article.

The predictions made here are a best-case scenario, where all particles conform to the assumption of spherical particles of homogeneous composition and a negative power-law size distribution. We suggest a definition for "optically significant" particle sizes, which are the sizes required to account for up to $95 \%$ of total scattering or backscattering. In spite of the restriction to a best-case situation, results from the numerical assessments conducted here indicate that the optically significant size range required for most marine particle populations is far wider than previously suggested. This is due to the sensitivity of optical properties to deviations from size distribution power-law exponents that are exactly equal to -4 . For shallow-size distribution slopes, approaching -3.2 , particles of millimeterlength scales must be considered in order to properly account for the optical properties of the water.

To cover the range of size distribution slopes that have been observed in situ, it is necessary for measurements and numerical predictions to include (or at least consider the effects of) particle sizes that span $0.05-2000 \mu \mathrm{m}$. Furthermore, the impact of these particle size-related effects should be considered in the design of instrumentation for IOP measurements and interpretation of data obtained with such instrumentation. Measurements of IOP should be performed with instrumentation capable of analyzing these particle sizes without disruption to ensure that large particles are accurately accounted for.

This work was supported by an NERC grant (NE/ H021293/1) and an award of a NERC Advanced Fellowship (NE/E013678/1) to D. McKee. The authors would also like to thank W. Slade for his "Fastmie" Mie theory code and three anonymous reviewers who have provided helpful comments on the manuscript.

\section{References}

1. D. Eisma, "Flocculation and de-flocculation of suspended matter in estuaries," Netherlands J. Sea Res. 20, 183-199 (1986).

2. T. G. Milligan, "Particle (floc) size measurements with benthos 373 plankton silhouette camera," J. Sea Res. 1, 93-100 (1996).

3. R. A. Reynolds, D. Stramski, A. M. Wright, and S. B. Wozniak, "Measurements and characterization of particle size distributions in coastal waters," J. Geophys. Res. 115, C08024 (2010).

4. G. W. Graham, E. J. Davies, W. A. M. Nimmo-Smith, D. G. Bowers, and K. M. Braithwaite, "Interpreting LISST-100X measurements of particles with complex shape using digital in-line holography," J. Geophys. Res. 117, C05034 (2012).

5. D. McKee and A. Cunningham, "Identification and characterization of two optical water types in the Irish Sea from in-situ inherent optical properties and seawater constituents," Estuar. Coast. Shelf Sci. 68, 305-316 (2006).

6. G. Chang and A. L. Whitmire, "Effects of bulk particle characteristics on backscattering and optical closure," Opt. Express 17, 2132-2142 (2009).

7. D. Risović, "Two component model of the sea particle size distribution," Deep Sea Res. Part I 40, 1459-1473 (1993).

8. M. Jonasz and G. Fournier, "Approximation of the size distribution of marine particles by a sum of log-normal functions," Limnol. Oceanogr. 41, 744-754 (1996).

9. D. Stramski and D. A. Kiefer, "Light scattering by microorganisms in the open ocean," Prog. Oceanogr. 28, 343-383 (1991).

10. M. Babin, A. Morel, V. Fournier-Sicre, F. Fell, and D. Stramski, "Light scattering properties of marine particles in coastal and open ocean waters as related to the particle mass concentration," Limnol. Oceanogr. 48, 843-859 (2003).

11. M. Jonasz and G. R. Fournier, Light Scattering by Particles in Water: Theoretical and Experimental Foundations (Elsevier Academic, 2007). 
12. W. H. Slade and E. Boss, "Calibrated near-forward volume scattering function obtained from the LISST particle sizer," Opt. Express 14, 8, 3602-3615 (2006).

13. C. F. Bohren and D. R. Huffman, Absorption and Scattering of Light by Small Particles (Wiley, 1983).

14. E. Aas, "Refractive index of phytoplankton derived from its metabolite composition," J. Plankton Res. 12, 2223-2249 (1996).

15. H. C. van de Hulst, Light Scattering by Small Particles (Dover, 1955).
16. A. Khelifa and P. S. Hill, "Models for effective density and settling velocity of flocs," J. Hydraul. Res. 44, 390-401 (2006).

17. E. Boss, W. H. Slade, and P. S. Hill, "Effect of particulate aggregation in aquatic environments on the beam attenuation and its utility as a proxy for particulate mass," Opt. Express 17, 9408-9420 (2009).

18. G. Dall'Olmo, T. K. Westberry, M. J. Behrenfeld, E. Boss, and W. H. Slade, "Significant contribution of large particle to optical backscattering in the open ocean," Biogeosciences 6 , 947-967 (2009). 